\title{
Las prácticas educativa y docente en un grupo de profesores universitarios
}

\author{
Educational and teaching practices \\ in a group of university professors
}

Maria Isabel Pérez Ornelas*

\begin{abstract}
RESUMEN
Se problematiza la acción del profesorado para caracterizar las prácticas docente y educativa al nivel superior, a fin de hacer visible lo que sucede en el aula. Por ello, se tematiza sobre estos dos tipos de práctica, desde su acepción más simple hasta la apoyatura teórica. Finalmente, se articula la discusión conceptual con un grupo de maestros de la Facultad de Economía de la UNAM; en este sentido, se describen los hallazgos de su análisis.
\end{abstract}

Palabras clave: profesorado universitario, práctica docente y educativa

\section{ABSTRACT}

The action of higher education professors is problematized so as to characterize their teaching and educational practices, in order to bring what happens in the classroom to light. These two types of practices are discussed, from their simpler to their more theoretical connotations. Finally, the article reports on the findings obtained from a conceptual discussion on the subject held with a group of professors from the Department of Economics at the National Autonomous University of Mexico.

Key words: University faculty, teaching and educational practice

\section{INTRODUCCIÓN}

El presente documento tiene el propósito de visibilizar la práctica docente y educativa de un grupo de profesores y profesoras universitarias. Para esto, primero se plantea el problema y se expone el interés sobre la labor docente de este nivel educativo. Posteriormente, se tematiza sobre el sentido y el significado de las prácticas educativa y docente, recurriendo a autores que permiten situarlas. En un tercer momento, se contextualiza el análisis de dichas prácticas, en este caso concreto, en la Facultad de Economía de la Universidad Nacional Autónoma de México (UNAM), y se caracteriza cómo es la acción educativa del grupo de maestros y maestras, tanto al nivel macro (práctica educativa), como micro (práctica docente). Por último, se ofrecen algunas reflexiones que

\footnotetext{
*Centro de Estudios Educativos, Universidad Nacional Autónoma de México; issabellula@hotmail.com
} 
dan cuenta de la importancia de recuperar el quehacer cotidiano de este actor educativo.

\section{PLANTEAMIENTO DEL PROBLEMA}

El interés en el profesorado de educación superior tiene que ver con mi área profesional como pedagoga y con mi cercanía con grupos de profesores y profesoras en procesos de formación docente, de los que he sido compañera y formadora. Como juez y parte, comparto situaciones problemáticas del gremio, algunas conocidas o visibilizadas, como las condiciones laborales; no ser "maestros y maestras" de formación inicial; exigencias intrínsecas de la educación actual, como la actualización y la formación constantes. Por lo anterior, considero pertinente cuestionar sobre el mundo complejo en que vivimos, donde la realidad nos desborda en lo inmediato y nos impide ver, justamente, a ese actor educativo con saberes propios que habría que aprovechar, y no bombardear desde una pedagogía prescriptiva y arbitraria, como sucede, por lo general, en los programas de formación docente a los que se encuentran sometidos.

En términos concretos, en este artículo se problematiza la acción docente, en el sentido de caracterizar cómo son las prácticas docente y educativa en un grupo de profesores que ha participado en procesos formativos. La pregunta nodal que guía el desarrollo de este planteamiento radica en qué hacen las y los docentes universitarios en sus clases, con el propósito de visibilizar lo que sucede cotidianamente en las aulas de este nivel educativo, mediado por su concepto de educación.

\section{¿QUÉ SON LAS PRÁCTICAS EDUCATIVA Y DOCENTE?}

Ya que interesa situar la cotidianidad del profesorado universitario, tomando como referente sus prácticas docente y educativa, es pertinente tematizarlas; por ello es primordial dilucidar una diferencia de orden conceptual, pues aunque se podría suponer que tienen un mismo significado, se considera que cada término encierra uno distinto, que se entretejen en la función del cuerpo docente. 
Por lo anterior, primero es necesario ubicar el significado de práctica, para luego plantear un debate de sentido que permita mirarla como categoría construida para dar cuenta de la perspectiva docente y educativa. El término práctica proviene del latín practice y del griego praktikée. La Real Academia Española lo define como "los conocimientos que enseñan el modo de hacer algo", o "que piensa o actúa ajustándose a la realidad y persiguiendo normalmente un fin útil” (RAE, 2014). También se puede anotar acepciones como la que ofrece la Enciclopedia del idioma: "Ejercicio que, bajo la dirección de un maestro y por cierto número de años, tienen que hacer algunos para habilitarse y poder ejercer públicamente su profesión" (Alonso, 1998: 3371).

Desde el marco de la educación, la práctica didáctica adquiere significado como la encargada de "enlazar el conocimiento teórico del fenómeno educativo en el aspecto de la relación docente-discente con el futuro ejercicio profesional. En el fondo de toda práctica hay siempre un hacer" (Labor, 1970: 727).

En este sentido, es pertinente de preguntarse qué son práctica educativa y práctica docente; para responder, se ha realizado una búsqueda en estudios que problematizan la formación docente en educación y, particularmente, en educación superior.

\section{La práctica educativa}

Benilde García Cabrero y sus colaboradores entienden la práctica educativa como:

... el conjunto de situaciones enmarcadas en el contexto institucional y que influyen indirectamente en los procesos de enseńanza y aprendizaje propiamente dichos; se refiere a cuestiones más allá de las interacciones entre profesores y alumnos en el salón de clases, determinadas en gran medida, por las lógicas de gestión y organización institucional del centro educativo (2008: 3-4).

Asimismo, señalan que la práctica educativa, al referirse a factores contextuales, es una "actividad dinámica, reflexiva, que debe incluir la intervención pedagógica ocurrida antes y después de los procesos interactivos en el aula" (ibid.: 4, cursivas mías). En 
este sentido, interesa el denominado triángulo interactivo: docente, alumno, contenido (véase Coll, 1997 y 2010), si bien se considera pertinente agregar la evaluación, ya que el proceso enseñanza-aprendizaje no se reduce a los aconteceres lineales en el aula; además, es de suma importancia lo que realiza el docente fuera de esta, como planear los contenidos programáticos, idear estrategias de enseñanza y aprendizaje, entre otras cuestiones, de manera explícita o implícita.

\section{La práctica docente}

El mismo grupo de estudiosos describe la práctica docente como "el conjunto de situaciones dentro del aula, que configuran el quehacer del profesor y de los alumnos, en función de determinados objetivos de formación circunscritos al conjunto de actuaciones que inciden directamente sobre el aprendizaje de los alumnos" (ibid:: 4).

Otro autor que habla sobre esta práctica, desde una postura crítica, es Freire, quien señala que debe estar

... implícita en el pensar acertadamente, encierra el movimiento dinámico, dialéctico, entre el hacer y el pensar sobre el hacer. El saber que indiscutiblemente produce la práctica docente espontánea o casi espontánea, 'desarmada', es un saber ingenuo, un saber hecho de experiencia, al que le falta rigor metódico que caracteriza a la curiosidad epistemológica del sujeto (2009: 39-40).

Para Freire, la práctica docente es esencialmente humana, formadora y ética en el quehacer.

Para Cayetano de Lella la práctica docente puede entenderse:

... como una acción institucionalizada y cuya existencia es previa a su asunción por un profesor singular. Frecuentemente se concibe la práctica docente como la acción que se desarrolla en el aula y, dentro de ella, con especial referencia al proceso de enseñar... El mundo de las prácticas permite revisar los mecanismos capilares de la reproducción social y el papel directo o indirecto del docente crítico en la conformación de los productos sociales de la escuela (1999). 
Entonces, la práctica educativa es mirar, de manera "macro", el proceso de enseñanza-aprendizaje, mientras que la práctica docente da cuenta de las interacciones que propicia el profesor o profesora en relación con la enseñanza; además, se considera que estas no solo se producen en el aula como espacio privilegiado. También importa reconocer el diálogo permanente entre las diferentes prácticas, como señala De Lella: "es claro que existe una fuerte interacción entre práctica docente, institución escolar y contexto, ya que la estructura global del puesto de trabajo condiciona las funciones didácticas que se ejercen dentro del mismo" (1999).

Como se aprecia, ambas definiciones se intersectan en el quehacer del docente; por ello, se considera significativo que en la educación superior el profesor o profesora cuente, también, con una formación didáctico-pedagógica, a fin de que responda a las necesidades educativas de los futuros profesionistas que el país requiere, frente a las problemáticas de este mundo globalizado.

De esta suerte, la categoría nodal en este trabajo es práctica, adjetivada con dos palabras que condensan una multiplicidad de significados: docente y educativa, y que por ello se articula con el habitus de Bourdieu, ya que "la práctica es producto de un habitus que es, en sí mismo, resultado de la incorporación de las regularidades y tendencias inmanentes del mundo, contiene en sí una anticipación de estas tendencias y regularidades" (1995: 95).

Si se parte de la aseveración, junto con este autor, de que el habitus es una estructura estructurante estructurada, se puede ver que las prácticas docente y educativa de las y los profesores son modificables, en tanto que:

La actividad práctica, en la medida en que tiene un sentido, en que es sensata, razonable, es decir, generada por habitus ajustados a las tendencias inmanentes del campo, trasciende el presente inmediato por medio de la movilización práctica del pasado y la anticipación práctica del futuro inscrito en el presente en un estado de potencialidad objetiva... la teoría de la práctica condensada en las nociones de campo y habitus permite descartar la representación metafísica del tiempo y la historia como realidades de sí mismas, externas y anteriores a la práctica (ibid.: 95). 
Por lo anterior, importa recuperar la práctica en la acción docente de las y los profesores universitarios, ya que se considera que las tematizaciones anteriores no profundizan en las dos prácticas, es decir, son tematizaciones generales que no permiten situar qué hace el profesorado y cómo ejerce su docencia universitaria, esto es, su habitus.

\section{LAS PRÁCTICAS EDUCATIVA Y DOCENTE EN PROFESORES UNIVERSITARIOS}

Por el interés, como se ha dicho, de ubicar la cotidianidad de las y los docentes universitarios, se realizó la investigación cualitativa "La práctica educativa y docente de profesores universitarios y su relación con procesos formativos. Aportes al debate". ${ }^{1} \mathrm{Al}$ tratarse de un estudio de caso, donde importa "la particularidad y... la complejidad, para llegar a comprender su actividad en circunstancias importantes" (Stake, 1998: 11), se buscó el detalle de la práctica docente de los maestros participantes en sus contextos como personas y profesionales en economía y en la enseñanza.

De esta forma, se recurrió a la etnografía y a la teoría fundamentada como herramientas de análisis. La primera puntualiza la necesidad de una descripción densa, que implica el análisis de las múltiples relaciones que se entretejen para dar sentido y significado a lo observado (Geertz, 1987: 22). Dada la intención de observar a los participantes en su cotidianidad educativa, se recurrió a la observación participante con notas de análisis. ${ }^{2}$ Yuni y Urbano señalan que la etnografía es un enfoque metodológico que ayuda

\footnotetext{
${ }^{1}$ Esta investigación se realizó en el marco de la Maestría en Desarrollo y Planeación de la Educación, en la Universidad Autónoma Metropolitana-Xochimilco, con apoyo de Conacyt y con la asesoría de la Dra. Sonia Comboni Salinas, profesora titular de tiempo completo en esta Universidad.

${ }^{2}$ La observación participante consiste en "captar la realidad social y cultural de una sociedad o grupo social determinado, mediante la inclusión del investigador en el colectivo objeto de estudio" (Fernández, 2009: 165-166). Cabe destacar que la observación que se llevó a cabo fue solo de una clase con videograbadora y audiograbadora, y se ha denominado no participante, porque no se tuvo interacción con las actividades, aunque se reconoce que la presencia modificó la dinámica cotidiana, principalmente en el alumnado, y en algunos casos en las y los maestros. Sin embargo, de acuerdo con Bertely, las modificaciones, más que considerarse como inferencias, deben valorarse como datos significativos (2000: 49). Como esta herramienta se toma de la etnografía, se utilizaron los instrumentos de análisis: registro de clase, secuencia de clase, segmentos y caracterización (Pérez, 2013).
} 
al investigador a describir e interpretar las interacciones de las personas, por lo que su principal contribución es "aportar datos descriptivos valiosos de los contextos, actividades y creencias de los participantes en los escenarios educativos" (2005: 111). Esto permite inducir la cultura de una institución educativa.

Asimismo, se consideró pertinente utilizar algunos elementos de la teoría fundamentada para construir categorías en el proceso de observación de las prácticas referidas. Esta metodología cualitativa permite fundamentar los conceptos, por ello se consideró necesaria para teorizar, desde el empirismo, qué acciones nutren a la práctica docente y cuáles a la práctica educativa. Así, el muestreo teórico ${ }^{3}$ se fue pensando a través de los avances de las observaciones y de la indagación (Strauss y Corbin, 2002) y quedó constituido por un grupo de profesores que reunía las siguientes características:

- Ser economista de formación inicial (licenciatura).

- Haber participado en cursos y proyectos orientados a su formación como docente.

- Aceptar ser entrevistado y observado en sus clases.

De una gama de 16 docentes con diversos perfiles y nombramientos, ${ }^{4}$ solo se trabajó con seis (cuatro mujeres y dos hombres, que van desde los cinco hasta los 24 años de antigüedad en la docencia).

Cabe señalar que, en este estudio, de acuerdo con Taylor y Bogdan, "en el muestreo teórico, el número de 'casos' estudia-

\footnotetext{
${ }^{3}$ Se recurrió a la técnica de la entrevista por el interés de "comparar la información obtenida, tratando de dar una denominación común a un conjunto de datos que comparten una misma idea" (Soneira, 2006: 156-157). Esta comprende tres ejes: 1) trayectoria docente, 2) percepción de la docencia, y 3) formación docente. Para la construcción del guion se tuvo presente la trayectoria de vida; por ello, el primer eje está orientado a ¿cómo se hizo docente? y ¿por qué continuar en la docencia? Es importante enfatizar que, a pesar de recurrir a las aportaciones de la teoría fundamentada, también se partió de la categoría de habitus de Bourdieu, porque se considera que los profesores tienen una historia incorporada que les ha permitido desarrollar su docencia por intuición.

${ }^{4}$ Ocho profesores ordinarios de asignatura y dos definitivos; dos profesores de carrera y tiempo completo; dos ayudantes de profesor; un técnico académico y un profesor sin remuneración por parte de la institución. Dos de los participantes tienen como formación inicial Ingeniería Química Metalúrgica e Ingeniería Química por la Facultad de Química de la UNAM; uno señaló que tiene dos licenciaturas: Ingeniería y Economía, los demás estudiaron Economía.
} 
dos carece relativamente de importancia. Lo importante es el potencial de 'caso' para ayudar al investigador en el desarrollo de comprensiones teóricas sobre el área estudiada de la vida social" (1990: 108).

Tras la breve descripción de la investigación, en las siguientes líneas se expone lo que se encontró con respecto a las prácticas docente y educativa.

\section{¿CÓMO ES LA PRÁCTICA EDUCATIVA DE LAS Y LOS DOCENTES UNIVERSITARIOS?}

Para articular lo tematizado sobre la práctica educativa -entendida como la concepción que se tiene sobre la educación y la docencia en un nivel macro y que implica factores contextuales, donde importa la reflexión y el ajuste pedagógico que pueda llevar a cabo el profesor, antes y después de la práctica en el aula-, se han encontrado, con la apoyatura de la teoría fundamentada y con los actores educativos, las siguientes categorías de análisis e interpretación que nutren la conceptualización inicial: percepción sobre la docencia, trayectoria docente, conceptualización del alumnado y relación pedagógica.

\section{Percepción sobre la docencia}

La percepción sobre la docencia es la manera de comprender y dar sentido al quehacer docente. Se puede reflejar en la apreciación sobre el proceso de enseñanza-aprendizaje, los aspectos positivos y negativos alrededor de este. Estos indicadores se describen a continuación.

La docencia para las y los maestros tiene connotaciones diferentes; la perciben como colaboración en el proceso de enseñanza-aprendizaje, una profesión, un arte, una disciplina, una vocación y como una transmisión de conocimientos. Un punto común de dos testimonios es que la aprecian como disciplina que requiere un trabajo formal y sistemático: "Una disciplina que requiere actualización permanente" (Cuest.D/F3), mientras que dos maestras la ven como arte y vocación, lo cual disminuye su carácter profesionalizante y fortalece el de la vocación, entendida 
como la "Inspiración con que Dios llama a algún estado, especialmente al de religión" 5 (RAE, 2014).

Una cuestión a destacar es que la mitad de las profesoras y los profesores manifiesta, de manera implícita, que la docencia es un proceso en el que se involucran docente y alumnado: "Colaborar en el proceso de enseñanza-aprendizaje" (MEM1); "Mucho compromiso y responsabilidad para compartir conocimientos" (MEF3). También que solo en un caso está explícito el estudiantado: "es una gran responsabilidad de transmitir y hacer que los alumnos se hagan de conocimientos para su formación profesional y humana" (MEM2).

El sentido ${ }^{6}$ que otorgan a ser docentes en educación superior está relacionado, mayoritariamente, con el docente como persona y profesionista, por ejemplo: "Un mayor compromiso para seguirme preparando y ser mejor cada día” (MEF4); cabe señalar que estas respuestas son de las profesoras, mientras que dos participantes, que corresponden a los profesores, lo relacionan más con la formación profesional e integral del grupo de estudiantes: "Es la formación integral de los futuros profesionistas" (MEM1).

Con respecto a cómo definen el proceso de enseñanza-aprendizaje, se tiene que la mayoría de docentes lo representan como un proceso impersonal, ya que no refieren si hay un destinatario ni en la enseñanza ni en el aprendizaje; por ejemplo, describen que es un "Proceso de transformación" (MEF4). En este sentido, casi la mitad de ellos recupera el conocimiento como parte de dicho proceso; esto se puede ubicar en las siguientes anotaciones: "Como un proceso en el que se comparten saberes, conocimiento, y que implica una disposición total para reaprender constantemente" (MEF1).

Los aspectos positivos y negativos que los y las docentes observan en su práctica adquieren matices interesantes porque, por un lado, atribuyen lo positivo a su persona, mientras que lo negativo, a factores externos.

\footnotetext{
${ }^{5}$ Para esta construcción, el término vocación que explica más el comportamiento de las personas que participaron en la investigación es el expuesto; no obstante, se reconoce que vocación también puede tener otras acepciones, como la de invocar, esto es, llamar o hacer algo y dedicar la vida a aquello para lo que uno siente tener aptitudes y capacidades.

${ }^{6} \mathrm{La}$ RAE lo define como el "Modo particular de entender algo, o juicio que se hace de ello" (2001).
} 


\begin{tabular}{|l|l|}
\hline \multicolumn{1}{|c|}{ Aspectos positivos } & \multicolumn{1}{c|}{ Aspectos negativos } \\
\hline & $\begin{array}{l}\text { 1. Cuestiones institucionales; laborales y curriculares. } \\
\text { Institucionales: infraestructura. }\end{array}$ \\
$\begin{array}{l}\text { 1. El aprendizaje como primer elemento del quehacer } \\
\text { docente (colaborativo y constante). }\end{array}$ & Laborales: formas de contratación. \\
2. Las estrategias de enseñanza-aprendizaje como & Curricular: múltiples enfoques teóricos y tiempos. \\
recurso que apoya el desarrollo de los temas. & 2. Cuestiones relacionadas con el alumnado. \\
3. La cuestión personal para desarrollar la docencia. & Desempeño escolar y actitud ante el estudio. \\
4. Los elementos didácticos. & Recursos económicos. \\
5. La preparación como profesores. & 3. Profesionales de la práctica docente. \\
& Dominio de la asignatura. \\
& Falta de formación pedagógica. \\
\hline
\end{tabular}

\section{Trayectoria docente}

La trayectoria es un indicativo de la percepción del profesorado sobre esta profesión, debido a que es un reflejo de las acciones que ha realizado para permanecer en la docencia. Por ello es importante situar el curso seguido; esto se puede explicitar mediante los motivos para involucrarse inicialmente con la enseńanza y las motivaciones para continuar en el gremio. Interesa la trayectoria en el plano formal institucional.

Del grupo participante, cuatro manifestaron entre los motivos para involucrarse con la docencia el gusto por esta profesión; los restantes reflejan, ya sea su buen desempeño escolar o un aspecto político-académico de la década de los setenta, fecha en que se dio la expansión de la universidad pública en México.

Una cuestión nodal que, de cierta manera, ha marcado la trayectoria de las y los participantes, es que tuvieron su primer acercamiento con la docencia por una selección del profesorado titular para ser ayudante de profesor. ${ }^{7}$ La selección puede ser arbitraria, en el sentido de que, a partir de su experticia, el titular recurre a un primer filtro que, desde nuestra perspectiva, es el reconocimiento de las habilidades del estudiantado para preservar el gremio docente. Hemos denominado a esto, en primera instancia, capital social, en el sentido bourdiano: la docencia

\footnotetext{
${ }^{7} \mathrm{El} \mathrm{rol} \mathrm{que} \mathrm{desempeña} \mathrm{el} \mathrm{ayudante} \mathrm{de} \mathrm{profesor} \mathrm{está} \mathrm{ligado} \mathrm{con} \mathrm{la} \mathrm{intencionalidad} \mathrm{del} \mathrm{titular;}$ es un proceso formativo desde la misma práctica docente. Al respecto, el grupo de participantes, señala que, parte de sus labores, consistía en el trabajo operativo de la clase, es decir, revisar tareas o trabajos, pasar asistencia, etc., además de participar esporádicamente en las sesiones e incluso quedarse a cargo del grupo por ausencia del titular.
} 
como gremio que busca perpetuarse, porque el maestro, experto, reconoce al otro y lo proyecta para formar parte de la academia, "el maestro me daba la oportunidad de que yo le enseńara a mis propios compañeros...” (Ent./ME5:4).

Al tener su primer acercamiento a la docencia como ayudante de profesor, importa indagar qué los/las motivó a permanecer en ella; el punto articulador de este grupo son los beneficios que puede aportar al sujeto estar en la enseñanza como docente, desde la permanencia en un cargo administrativo, la actualización, la consolidación de conocimientos, la transformación y la superación, el gusto por comunicarse con otras personas, hasta tener asegurada una fuente de empleo y, por ende, un ingreso monetario. Otra cuestión interesante es que el alumnado está en segunda mención; esto se observa porque solo dos docentes lo explicitan: "La transformación y la superación primero en mí y posteriormente en los alumnos" (MEF4).

Al permanecer en la docencia, se espera ser el titular de la asignatura; por ello, en la trayectoria también es necesario poner atención en el proceso para ser profesor titular, independientemente del tipo de nombramiento y contrato. ${ }^{8}$ Se tiene que cinco del grupo de docentes participantes fueron invitados por la institución, con algunas variantes, mientras que el resto tuvo que participar en la convocatoria por concurso de oposición. Cabe destacar que, de los cinco casos que fueron invitados, a dos se les requirió para sustituir a los profesores titulares por diversas causas, mientras que uno tuvo que estar "en la banca, esperando a que [le] dieran una oportunidad" (Ent./ME3:2). En lo que respecta a la profesora que presentó concurso para la asignación de la materia, señala: "cuando llegó el momento de la titularidad, yo ya tenía dos cosas: por un lado, tenía mis tres años de haber trabajado como adjunta y, por otro, ya estaba titulada" (Ent./ME8:5).

En el trayecto docente se puede ubicar que este grupo ha mantenido la misma línea de estudio, pues han estado involucrados desde ayudantes de profesor, por lo menos, con el contenido

\footnotetext{
${ }^{8} \mathrm{El}$ personal académico de la UNAM está integrado por Técnicos académicos, Ayudantes de profesor o de investigador y Profesores e investigadores, con el nombramiento interino o definitivo o por contrato de prestación de servicios, de acuerdo con los art. $4^{\circ}$ y $5^{\circ}$ del Estatuto del Personal Académico (EPA).
} 
de una asignatura; la única variante que se encuentra en cinco de los casos es el cambio de nombre de la materia, que se debe a modificaciones de los planes de estudios correspondientes. Lo anterior indica que hay una especialización de la línea de estudio.

\section{Conceptualización del alumno}

Se ha encontrado que la forma de conceptualizar al alumno puede determinar el tipo de relación pedagógica que se establece en y fuera del salón de clases. En el marco de esta investigación, se encuentra que el profesorado conceptualiza al estudiante como aquel/aquella persona que es responsable de su aprendizaje y es guiado por el/la docente. Un estudiante activo permite al docente aprender y comprender mejor la temática enseńada, así como motivar y diversificar estrategias de enseñanza-aprendizaje.

Dos docentes señalan que se aprende del alumnado cuando se está dispuesto; por ejemplo, una profesora comenta: "cuando escuchamos o leemos a los muchachos, vamos aprendiendo cosas que nos hacen reflexionar sobre lo que estamos dando" (Ent./ ME8:16). En esta línea, la mayoría señala que depende del profesorado que el alumnado aprenda ya que, actualmente, son una guía para el aprendizaje; al respecto, un profesor refiere "hay investigaciones que te demuestran que un estudiante va aprender cuando tú lo ayudas o utilizamos simuladores" (Ent./ME7:13).

Es importante el hecho de que las y los docentes conciban al alumnado como un actor activo en el proceso de aprendizaje, porque reconocen cómo aprende, y su acción educativa no se centra únicamente en el proceso de enseñanza. $\mathrm{Al}$ respecto vimos que la mitad de los participantes ubica que las habilidades para aprender dependen de las capacidades de los hemisferios cerebrales; en este sentido, dos de ellos señalan que el aprendizaje se da a través de los canales de percepción dominantes: ${ }^{9}$ que son el visual, el auditivo y el kinestésico; uno refiere que "hoy día, los estudiantes privilegian más el lado derecho de su cerebro, que es la parte visual" (Ent./ME7:13).

\footnotetext{
${ }^{9}$ Entre las teorías neurocientíficas se encuentran los canales de percepción, que indican la manera en que se percibe, organiza y expresa la información; puede ser visual, auditiva o kinestésica. Cabe aclarar que estos temas han sido tratados en algunos módulos que se ofrecieron en el Diplomado de Habilidades Docentes.
} 
La mitad restante, si bien tiene en común que el aprendizaje también depende del estudiantado, va más allá en sus conceptualizaciones de la percepción cerebral; tal es el caso de una profesora que argumenta que somos seres integrales, "tanto alumnos como maestros debemos estar bien para poder interactuar adecuadamente" (Ent./ME8:22-23); además, agrega que "Un muchacho que es maltratado por el maestro, pues no va a querer llegar a esa clase" (Ent./ME8:22).

Otro aspecto a destacar es que el grupo de profesores comprende cuán importante es desarrollar, en el alumnado, "diferentes habilidades sobre un mismo conocimiento" (Ent./ME12:27) para que lo ancle desde diversas estrategias. Finalmente, interesa señalar que una docente considera que el educando es el que construye su saberes y se los apropia; a propósito, comenta: "Uno hace su conocimiento y ustedes [se refiere al grupo] tienen la obligación de trabajarlo, porque se maneja mucho esta idea de que hay una transferencia de conocimiento de parte del docente hacia el alumno y eso no es cierto" (Ent./ME1:17).

\section{Relación pedagógica}

Dado el contexto anterior se ubica, también, el tipo de relación pedagógica que establecen las y los docentes con el grupo de alumnos y alumnas en el contexto educativo; en este sentido, se considera pertinente puntualizar que por esta se entiende la interacción y la comunicación que se construye entre los actores en el proceso enseñanza-aprendizaje, en un escenario educativo donde se potencia, en términos vygostkyanos, la interrelación entre los conocimientos externos que se deben adquirir y la capacidad o el proceso intrapsicológico de los aprendizajes, además de la comunicación verbal intencionada, de acuerdo con Ausubel, para propiciar un aprendizaje significativo ( $c f r$. Vygotsky, 1979, 1995; Ausubel, 1976).

Con lo anterior cobran interés las siguientes categorías que nutren la conceptualización realizada en este documento de relación pedagógica: interacción, encuadre del curso, además del tipo de comunicación verbal y no verbal.

Tras lo anterior, se encuentra que cinco de las y los docentes recuperan la importancia de la interacción con el estudiantado, 
bien sea para conocer si están aprendiendo a través de gestos y exclamaciones, bien mediante pláticas directas en las sesiones. Para ilustrar esto se tiene que, para un profesor: "trabajar con jóvenes y ver que de repente descubren algo o algo les queda por fin claro. Cuando te dicen: 'ah' o truenan los dedos de, 'ah ya', o ese tipo de reacciones son muy satisfactorias” (Ent./ME7: 11-12).

Resulta significativo describir ${ }^{10}$ cómo se establece la comunicación pedagógica en el salón de clases, que son las relaciones sociales o interacciones entre el o la estudiante y el profesor, el grupo y este y el estudiantado en su conjunto.

En este sentido, se puede situar que las y los docentes establecen comunicación de manera intencionada (verbal o explícita) y de manera implícita (no verbal) con el grupo, en varios momentos de la sesión. En el análisis de las clases observadas se encontraron los siguientes actos comunicativos: conoce a los alumnos y las alumnas por su nombre; el salón de clases es un espacio que le permite movilidad; mira al estudiantado en las explicaciones; pregunta y da oportunidad de que respondan (no pasa a otro $\mathrm{u}$ otra; orienta las respuestas o, bien las corrige); orienta al alumnado en la actividad; utiliza el material didáctico como recurso, no como fin de su explicación; fomenta la participación; se interesa por el estudiantado (en lo personal, lo laboral y en aprendizajes), y responde a las dudas.

En la comunicación intencionada, un aspecto claro es el encuadre de la sesión, pues la mayoría de los participantes explica al grupo el tema o los temas que abordarán durante la clase; no obstante, tiene variantes que se enuncian a continuación: cinco de ellos inician la clase señalando el grupo los temas que se van a desarrollar; el restante no realiza introducción e inicia la asesoría preguntando si leyeron el material y comienza a preguntar. Tres de los cinco que introducen la clase, además de señalar el contenido, hacen un repaso de lo que abordaron en la clase anterior, mientras que uno también pregunta por la tarea. Otro aspecto a

\footnotetext{
${ }^{10}$ Para ello, se recupera información de la observación realizada, puntualmente, de una sesión en que los profesores desarrollaron, de la manera más natural posible, el tema correspondiente. Cabe agregar que, con cinco participantes, se usaron los siguientes instrumentos para el análisis de las sesiones: registro de clase con base en la videograbación; secuencias, segmentos y descripción de las clases; de la sexta participante, solo se tiene la observación y las notas de campo.
} 
destacar es que la mayoría de las y los profesores anota en el pizarrón los temas a abordar durante la clase, o bien, usa diapositivas.

Un acto comunicativo intencionado está constituido, en este caso, por las instrucciones que da el profesorado al grupo para realizar alguna actividad concreta, ya sea ejercicios en el cuaderno, pizarrón, manual o de tarea. $\mathrm{Al}$ respecto, se encuentra que depende del tipo de asignatura, pues algunas son teóricas, otras prácticas o, bien, son teórico-prácticas. En este sentido, se tiene que, del total, en algún momento de la sesión, cinco dan indicaciones para realizar ejercicios, y de estos, cuatro son de cálculo o representaciones de lo abordado en maquetas, pero destaca que dos de ellos brindan explicaciones claras sobre los procedimientos a seguir, que fungen como refuerzo en clase, además de que repiten los pasos y preguntan si hay dudas al respecto. Los dos restantes indican la actividad de manera general, lo que propicia que alumnos y alumnas pregunten varias veces qué van a hacer, gesto que interrumpe la secuencia de la clase; por ejemplo, un profesor señala que van a realizar una maqueta con plastilina y va estableciendo las instrucciones a lo largo de la actividad, por lo que se nota un desorden en el grupo.

También se ubica que las y los docentes participantes establecen una comunicación directa o dirigida en algunos momentos del desarrollo de la clase, ya sea con el grupo o con un alumno específico. Un aspecto que propicia, desde nuestra perspectiva, el grado de involucramiento que puede tener un profesor o una profesora con su grupo es si conoce el nombre de sus alumnos y alumnas; en este sentido, se tiene que únicamente dos maestros mencionan más de un nombre de algún o alguna estudiante en el transcurso de la clase.

Otra cuestión a destacar es la movilidad del docente en el salón de clase, pues se ubica que la mitad de las y los participantes utiliza el espacio del aula para desplazarse de un lado a otro, de tal forma que están más atentos a las actividades desarrolladas por el alumnado. La otra mitad limita sus traslados a puntos concretos en el aula, como es en la parte frontal o lateral, lo que de cierta manera les permite tener un control de todo el grupo, interponiendo una distancia. 
Finalmente, la importancia de ubicar el tipo de relación pedagógica que propicia el profesorado en la práctica docente, esto es, en el aula, permite situar su práctica educativa o sus concepciones sobre la enseńanza; además, se puede insinuar que, de cierta manera, también es un factor de motivación para su involucramiento activo en los procesos formativos que le permitan tener mayor conocimiento de los saberes pedagógicos y didácticos, pues se piensa que estar sometido a estos procesos de forma permanente implica que el habitus adquirido en sus años como estudiantes y ayudantes de profesor pueda modificarse a través de la reflexividad de su práctica.

\section{¿QUÉ HACEN LAS Y LOS DOCENTES EN EL AULA?}

Aquí se recupera la práctica docente de las y los participantes en la investigación, entendida como el actuar en el aula y los procesos implícitos o explícitos que se llevan a cabo en el desarrollo de la clase. Por ello, la definición inicial se puede establecer señalando las estrategias de enseñanza-aprendizaje, los recursos didácticos utilizados y, por ende, el diseño de materiales educativos, todo esto para propiciar el desarrollo potencial del estudiante.

\section{Estrategias de enseñanza-aprendizaje}

Para Díaz Barriga y Hernández las estrategias de enseñanza son "procedimientos que el agente de enseńanza utiliza en forma reflexiva y flexible para promover el logro de aprendizajes significativos en los alumnos" (2002: 141); por tanto, son medios o recursos para ajustar el apoyo pedagógico. En este sentido, tras el análisis de las clases observadas, se describen las estrategias de enseñanza-aprendizaje que utilizan las y los docentes participantes con mayor frecuencia, tanto al inicio y durante, como al final de la sesiones.

Al comienzo de la sesión, recurren a estrategias preinstruccionales ${ }^{11}$ como introducción al tema, aunque esta no siempre se da

\footnotetext{
${ }^{11}$ Las estrategias preinstruccionales son aquellas que "preparan y alertan al estudiante en relación con qué y cómo va a aprender” (Díaz y Hernández, 2002: 143). Además, propician la activación en el estudiantado, ya que se pueden recuperar saberes o conocimientos previos acerca del contenido a desarrollar.
} 
al inicio de la clase, en el sentido cronológico, ya que en algunas ocasiones se abarca más de un contenido. Se ubican dos tipos de estrategias: en uno, se recuperan los conocimientos previos del estudiantado, por parte del profesor y, en el otro, se realiza un recordatorio de lo revisado anteriormente a través de un resumen hecho ya por el docente, ya por el ayudante del profesor o por un alumno. En el primer caso, se tiene que solo dos de los profesores recuperan los conocimientos previos del grupo; de este, la mitad también recuerda el contenido visto con anterioridad o previo al actual para poder anclar el nuevo. Del segundo, se tiene que la mitad de las y los docentes acude al repaso de los elementos clave enseñados en sesiones anteriores.

Las estrategias que utilizan con más frecuencia las y los docentes, durante la sesión o co-instruccionales, de más a menos, son:

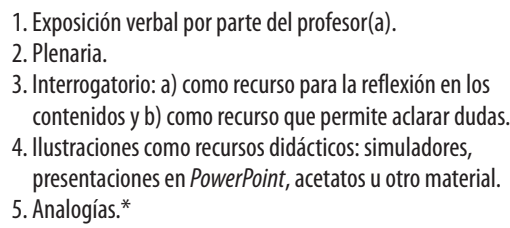

6. Ejemplos de la vida cotidiana para ilustrar el tema. 7. Resolución de ejercicios de manera individual y grupal (pizarrón, cuaderno, etcétera).

8. Trabajo en equipo.

9. Presentación pública del producto final, ya sea en el aula 0 en redes sociales.

* Las analogías son proposiciones que indican que una cosa 0 evento (concreto y familiar) es semejante a otro (desconocido y abstracto 0 complejo). (Díaz Barriga y Hernández, 2003: 142).

Como estrategias postinstruccionales, la mitad de las y los docentes recurre a tareas extra clase, como instrumento que permite reforzar y practicar lo revisado en las sesiones; por ejemplo, una docente enfatiza en una investigación en la que el alumnado aborde lo visto en el curso; dos profesoras acostumbran que sus estudiantes realicen ejercicios, ya que su materia es de matemáticas; entonces, para que practiquen fuera de clase, les piden elaborar tareas según el tema que han visto (MEF1, MEM1, MEF2, MEF3, MEF4).

Con lo anterior se sitúa que la práctica docente de las y los participantes de esta investigación obedece a diferentes ambientes de aprendizaje, ya que las estrategias de enseñanza-aprendizaje tienen impactos distintos que rebasan los alcances de este trabajo; no obstante, se puede enfatizar que cada uno de ellos las utiliza de acuerdo con el deber ser de su asignatura, mediado también 
por la perspectiva que tienen de esta; asimismo, se puede inferir que es un ajuste pedagógico no intencionado que, según Schön, es tácito y no reflexionado; esto se señala en el sentido de que imperan las clases expositivas, donde el principal interlocutor sigue siendo el o la docente $(1998,2008)$.

\section{Recursos didácticos}

Para apoyar la explicación del contenido que aborda en las clases, el grupo de docentes recurre a recursos didácticos conceptuales y materiales. Por ello, se consideró significativo visibilizar qué recursos utiliza y cómo es su tratamiento.

Es conveniente señalar que se coincide en que un recurso didáctico es un medio que utiliza o emplea "el docente para apoyar, complementar, acompañar o evaluar el proceso educativo que dirige u orienta" (Grisolía, s/f), diseńado o dirigido hacia el alumno con el propósito de que este aprenda de la mejor manera posible. En este sentido, se plantean dos tipos de recursos: los conceptuales y los materiales; los primeros tienen que ver con ejemplos, simulaciones, y los segundos, con instrumental como libros, texto, audiovisuales, proyector, etcétera.

En cuanto a los recursos didácticos materiales, como apoyatura a la explicación del profesorado, el más utilizado es el pizarrón, pues todo el grupo docente lo emplea en algún momento de la clase para hacer "anotaciones"; la mitad realiza esquemas, principalmente gráficas, debido al contenido teórico-práctico de la asignatura; por ejemplo, una maestra dibuja tablas con los datos a resolver por el alumnado como parte de la práctica.

La mitad recurre a recursos tecnológicos para apoyar el proceso de enseńanza-aprendizaje, y de estos, dos utilizan presentaciones en PowerPoint, mientras que el resto se auxilia de tecnología más avanzada y orientada al contenido de la asignatura en cuestión, como son los simuladores computacionales.

Un último recurso utilizado por un docente es la elaboración de maquetas con plastilina; el alumnado las realiza poniendo en práctica lo visto en clase. Un aspecto a destacar es la utilización de celulares inteligentes y de redes sociales en esta actividad, como parte del contexto juvenil actual. 
Referente a los recursos didácticos conceptuales, se encuentra que se apoyan con mayor frecuencia en ejemplos que les permitan ilustrar el contenido. Cinco docentes recurren a ejemplos durante el abordaje del tema, en distinta proporción; se destaca que solamente dos profesores los utilizan con mayor frecuencia; por ejemplo una maestra plantea casos hipotéticos de financiamiento, para que el alumnado realice las operaciones correspondientes.

Otro recurso conceptual es la técnica de interrogatorio, lo manejan en dos sentidos: uno, para motivar la participación del estudiantado, y otro para repasar el tema anterior. Es significativo hacer notar que cinco de las y los docentes apelan a esta técnica, pero tres de ellos lo utilizan como eje de la clase, ya que los cuestionamientos son dirigidos a que el estudiantado responda y realice las actividades.

Por último, se encuentra que la mayoría de los docentes, en algún momento de la clase, realiza un repaso del tema. Cabe subrayar que, por lo general, al inicio de la sesión se retoma lo abordado anteriormente y, en algunos casos, es un resumen de lo desarrollado durante la misma.

Si se recurre a Vygotsky $(1979,1995)$, se puede señalar que las y los docentes participantes intentan ajustar su ayuda pedagógica, a través de los recursos didácticos, a que el alumnado pase de la zona real a la zona potencial de aprendizaje. Asimismo, la mayoría de aquellos recurre al aprendizaje guiado, emplean con mayor frecuencia la plenaria -exposición verbal para toda la clase-; no obstante, es conveniente explicitar que se percibe que los recursos didácticos están más centrados en la enseñanza que en el aprendizaje del alumnado, por la forma en que son manejados por los y las docentes en el desarrollo de la clase.

\section{Materiales didácticos: el diseño}

Como se mencionó líneas arriba, cuatro de las y los docentes usan apoyos didácticos, aparte del pizarrón, para el desarrollo de su clase; la mitad de ellos diseña presentaciones en PowerPoint (diapositivas) con el contenido a tratar; otro docente usa simuladores computacionales y el resto prepara acetatos. 
Un aspecto a destacar es que dos de las maestras participantes diseñan ejercicios, que llevan impresos, ya sea en hojas blancas ya en acetatos, para que el grupo de estudiantes los realice, bien en la clase, bien como tarea, con el propósito de que practiquen lo visto; cabe señalar que las docentes que se auxilian de este recurso son las que imparten asignaturas de matemáticas en otro nivel educativo.

De los materiales que realizan las y los profesores podemos situar que, si bien la mayoría los diseña de acuerdo con la percepción de la asignatura (teórica, práctica o teórica-práctica) e indica el grado de involucramiento que tienen con la planeación y estructura de su clase, es importante destacar que estos no cumplen, de manera satisfactoria, con algunos lineamientos mínimos que deben poseer. Si seguimos a Ausubel, para que sean lógicos en su estructura, deben relacionar potencialmente el material de manera no arbitraria con la estructura cognoscitiva, además de procurar el significado psicológico, que tiene que ver con la "relacionabilidad intencionada y sustancial de las proposiciones lógicamente significativas con la estructura cognoscitiva de un alumno en particular lo que las hace potencialmente significativas para este" (1976: 64).

Lo anterior se menciona porque, de acuerdo con las características de los materiales utilizados, si bien la mayoría maneja el lenguaje técnico, propio de la economía, y en apariencia lleva una gradación del nivel y complejidad, entre otros elementos a los que alude Ausubel, desde el punto de vista pedagógico y didáctico se percibe una saturación de información en algunos y en otros la visibilidad no es la más adecuada. En este sentido, el material está centrado en la enseñanza y no como potencial del aprendizaje, a excepción de los simuladores, que permiten la práctica del conocimiento.

Tras la descripción de la práctica docente, es imprescindible acotar que las y los profesores utilizan estrategias de enseñanza para abordar el contenido en la clase, pero su actuar mantiene una dosis de la escuela tradicional, en la que el maestro es el principal actor en los procesos de enseńanza-aprendizaje. Lo anterior se sitúa en las principales estrategias llevadas a cabo, en los recursos didácticos y en los materiales que diseñan, que siguen centrados en el docente como recurso que permite explicar la temática. 


\section{A MANERA DE CIERRE}

Este trabajo pretende caracterizar lo que hacen las y los docente universitarios, tomando un pequeño grupo de la Facultad de Economía de la UNAM. Si bien se reconoce que la muestra no reúne los criterios metodológicos de un estudio cuantitativo, sí es significativa en cuanto que es un estudio de caso, donde importa la singularidad y la investigación detallada del quehacer de los seis docentes, ya que en la práctica educativa y docente se reconfigura el sentido y el significado de ser profesor o profesora.

Con lo anterior, se puede situar que la práctica educativa es la concepción que se tiene de la educación y la docencia pero que, precisamente, se nutre del actuar cotidiano, donde importa qué se piensa de los procesos de enseńanza-aprendizaje; cómo se enfrentan aquellas situaciones que posibilitan u obstaculizan dichos procesos; cómo ha sido la trayectoria docente, permeada por un habitus incorporado, que potencia el modo de dar las clases. Además, es significativa la manera de conceptualizar al alumnado, pues de esto depende la relación pedagógica y didáctica que se puede establecer, en términos de favorecer o no los procesos ya mencionados, y no quedar en una mera transmisión del conocimiento.

Lo anterior puede determinar la acción en concreto en el aula, o la práctica docente, pues se ubica el tipo de estrategias que ocupa el o la docente universitaria para promover aprendizajes en el alumnado, el acompañamiento de recursos didácticos (conceptuales y materiales) y el diseño de los materiales. Todo esto como parte del diseño metodológico que permite, desde la perspectiva del profesorado, que el alumnado aprehenda los conocimientos de la mejor manera posible.

Finalmente, se puede decir que, para el campo de la formación docente, importa mirar más la acción co0tidiana del profesorado universitario, y no ubicarlo como un ente en abstracto que necesita una formación didáctica y pedagógica, para cubrir la ignorancia o las carencias de esta índole. Estamos convencidas de que, si reconfiguramos a este actor y se recuperan sus saberes tácitos, sus experiencias y preocupaciones desde y en la práctica, la formación del profesorado tendría alcances más significativos y, quizá, más duraderos y eficaces en la formación de los alumnos de las instituciones de educación superior. 


\section{REFERENCIAS BIBLIOGRÁFICAS}

Alonso, Martín. Enciclopedia del idioma. Diccionario histórico y moderno de la lengua española (Siglos XII y XX) etimológico, tecnológico, regional e hispanoamericano, tomo III N-Z, México, Aguilar, 1998.

Ausubel, D. Psicología educativa. Un punto de vista cognoscitivo, México, Trillas. Trad. Roberto Helier Domínguez, 1976.

Bertely, M. Conociendo nuestras escuelas. Un acercamiento etnográfico a la cultura escolar, México, Paidós, 2000.

Bourdieu, Pierre. "3. Habitus, illusio y racionalidad", en Respuestas por una antropología reflexiva, México, Grijalbo, 1995, pp. 32-38 y 79-99.

Coll, C. ¿Qué es el constructivismo?, Argentina, Magisterio del Río de la Plata, 1997.

Coll, C. "La Construcción del conocimiento en el marco de las relaciones interpersonales y sus implicaciones para el currículum escolar", en Aprendizaje escolar y construcción del conocimiento, México, Paidós, 2010, pp. 177-188.

De Lella, C. "Modelos y tendencias de la formación docente", en Desarrollo escolar, Perú, OEI, 1999. Disponible en http:// www.oei.es/cayetano.htm Fecha de acceso, mayo de 2012.

Díaz-Barriga F. y G. Hernández Rojas. Estrategias docentes para un aprendizaje significativo. Una interpretación constructivista, México, McGraw-Hill, 2002.

Fernández Poncela, A. M. La investigación social. Caminos, recursos, acercamiento y consejos, México, Trillas, 2009.

Freire, P. Pedagogía de la autonomía. Saberes necesarios para la práctica educativa, México, Siglo XXI Editores, 2009.

García Cabrero, B., J. Loredo y G. Carranza. "Análisis de la práctica educativa de los docentes: pensamiento, interacción y reflexión”, en Revista Electrónica de Investigación Educativa, 2008. Disponible en http://redie.uabc.mx/NumEsp1/contenido-garcialoredocarranza.html Fecha de acceso, mayo de 2012.

Geertz, C. "1. Descripción densa: hacia una teoría interpretativa de la cultura", en La interpretación de las culturas, México, Gedisa, 1987, pp. 19-40. 
Grisolía, M. "Qué es un recurso didáctico", Venezuela, Facultad de Humanidades y Educación-Universidad de los Andes Venezuela, s/f. Disponible en http://webdelprofesor.ula. ve/humanidades/marygri/index.php Fecha de acceso, diciembre de 2012.

Labor. Diccionario de Pedagogía, tomo II, G-Z, España, Labor, 1970. Pérez Ornelas, Ma. Isabel. "La práctica educativa y docente de profesores universitarios y su relación con procesos formativos. Aportes al debate del caso de la Facultad de Economía de la UNAM", Tesis, México, UAM, 2013.

RAE. Diccionario de la Lengua Española, Madrid, Real Academia de la Lengua Española, 2014. Disponible en http://lema. rae.es/drae/?val=pr\%C3\%A1ctica+ Fecha de acceso, julio de 2014.

Schön, D. El profesional reflexivo. Cómo piensan los profesionales cuando actúan, España, Paidós, 1998.

Schön, D. La formación de profesionales reflexivos. Hacia un nuevo diseño de la enseñanza y el aprendizaje en las profesiones, España, Paidós, 2008.

Soneira, A. J. "La teoría fundamentada en los datos (Grounded theory) de Glaser y Strauss", en I. Vasilachis de Gialdino (coord.). Investigación cualitativa, Barcelona, Gedisa, 2006, pp. 153-173.

Stake, R. E. "Introducción. Estudio intensivo de los métodos de investigación con estudio de caso", en Investigación con estudio de casos, trad. Roc Filella, Espańa, Ediciones Morata, 1998.

Strauss, A. y J. Corbin. Bases de la investigación cualitativa. Técnicas y procedimientos para desarrollar la teoría fundamentada, Colombia, Editorial Universidad de Antioquia, 2002.

Taylor y Bogdan. "La entrevista a profundidad", en Introducción a los métodos cualitativos, Buenos Aires, Paidós-Studio, 1990, pp. 100-132.

Yuni, J. y C. A. Urbano. "Supuestos teóricos y epistemológicos de la etnografía", en Mapas y herramientas para conocer la escuela. Investigación etnográfica e investigación-acción, Córdoba, Argentina, Editorial Brujas, 2005, pp. 107-118. 
Vygotsky, L. "Aprendizaje y desarrollo", en El desarrollo de los procesos psicológicos superiores, México, Crítica/Grupo Editorial Grijalbo, 1979.

Vygotsky, L. Pensamiento y lenguaje, España, Paidós, Serie Cognición y desarrollo humano, 1995.

\section{Instrumentos de investigación}

\section{Entrevistas}

Ent./ME1, comunicación personal, 25 de octubre de 2011.

Ent./ME7, comunicación personal, 28 de octubre de 2011.

Ent./ME5, comunicación personal, 31 de octubre de 2011.

Ent./ME8, comunicación personal, 5 de noviembre de 2011.

Ent./ME3, comunicación personal, 27 de octubre de 2011.

Ent./ME12, comunicación personal, 25 de noviembre de 2011.

\section{Cuestionarios}

MEF1, cuestionario, 22 de octubre de 2011.

MEM1, cuestionario, 17 de octubre de 2011.

MEF2, cuestionario, 12 de marzo de 2012.

MEF3, cuestionario, 27 de octubre de 2011.

MEF4, cuestionario, 19 de noviembre de 2011.

MEM2, cuestionario, 20 de octubre de 2011.

\section{Registros de clase}

MEF1, registros de clase, 5 de noviembre/2011.

MEM1, registros de clase, 8 de noviembre/2011.

MEF2, registros de clase, 8 de noviembre/2011.

MEF3, registros de clase, 8 de noviembre/2011.

MEF4, registros de clase, 29 de octubre/2011. 